\title{
Teaching of optics in the optics age
}

\section{A. Parasnis}

A. S. Parasnis, "Teaching of optics in the optics age," Proc. SPIE 2525, 1995 International Conference on Education in Optics, (13 October 1995); doi: $10.1117 / 12.224026$

SPIE Event: SPIE's 1995 International Symposium on Optical Science, SPIE. Engineering, and Instrumentation, 1995, San Diego, CA, United States 


\title{
Teaching of optics in the optics age
}

\author{
A S Pārasnis \\ Formerly Professor of Physics, Indian Institute of Technology Kānpur, India ${ }^{1}$
}

\begin{abstract}
The present Optics Age of science had not been foreseen by curriculum-makers in many countries. The result is that during the last 25 years when activity in science, engineering, technology and industry related to optics and lasers was exploding in the advanced countries optics was being de-emphasized in curricula in these countries. The consequence is that these countries including India ${ }^{2}$ have not played good enough a role. It is shown that the changes needed are more in attitude, pedagogic tools, terminology, and teaching methodology than in curricular contents. Examples: (1) The sequence of topics be changed, (2) The concept of coherence be introduced by considering thought experiments with different light sources; it is shown that the Michelson interferometer along with an HeNe laser can be used to introduce spatial as well as temporal coherence, (3) Modern terminology be used, (4) Crystal optics which is difficult be divorced from the concept of polarization which is simple; greater attention be paid to polarization helix than to polarization ellipse so that the confusion in "handedness" is eliminated, (5) Group velocity be introduced as related to stationary phase approximation, (6) Modern terms like irradiance, temporal, spatial, frequency spectrum, spatial frequency, etc be used extensively, and so on.
\end{abstract}

Keywords: optics education, optics teaching, new methodology

\section{INTRODUCTION}

The history of Physics is that of Optics. For curricular purposes optics refers to UV, visible, IR and, often, microwave regions of the em spectrum; in particular, the visible region because that is how human beings perceive their environment with remarkable acuity by way of frequency (colour) and irradiance ("intensity") ranges. A single photon can excite a retinal rod.

Till 40 years ago much optics used to be taught. With the progressive need to teach newer subjects the content of optics was reduced in many countries (e $g$ India) although elsewhere optics led the way. Nor were new optics and methodology introduced. Describing the progress of science and technology by the dominant component we now discern the optics age. The last fifty years were marked by the development of lasers, holography, information theory, communication theory, visual arts (TV, displays, 3D movies), nonlinear optics, fibre optics, optical computing. Today India uses the technologies which arose from these developments but she is nowhere near participating substantially in the optics age. No original achievement of international standard can be pointed out. This is because of an incorrect perception on the part of policy-makers who do not $d o$ optics. Some think that all of optics comes out of " $E \& M$ " courses, others feel that optics means instrumentation, yet others feel that optics is glorified technology. Such prejudices have always existed everywhere but this one has been detrimental to the teaching of optics as a separate discipline with its own structure, methodology and achievement. At the research level, however, in many institutions there is sufficient activity in optics but the number of students wanting to go into it is restricted because teachers pass on their prejudices to students. If countries like India are to take strides into the optics age it is essential to strike where it will be most effective, viz in academic institutions. It is noteworthy that Dirac in his Principles of Quantum Mechanics has taken an example from optics, viz polarization by birferingence, to introduce the concepts of probability and superposition ( $p p$ 4-7), and more recently Feynman has built his popular book QED (Quantum Electrodynamics, Penguin Books 1985) entirely around the optical concepts of phase and amplitude. Curricula seem selectively to have reduced optics and sufficient efforts are not going into the process of its rehabilitation. The purpose of this paper is to induce that process.

\footnotetext{
${ }^{1}$ Present address: Flat Nr 17 (5th floor), Chaitraban Residency Building "F”, Off ITI Road Aundh, PUNÉ 411007, MH, India

${ }^{2}$ Henceforth "India" will symbolize countries which have neglected Optics.
} 


\section{TEACHING OF OPTICS}

The problem with teaching new optics, or old optics newly, is that its foundations changed radically a long time ago: in 1885 with Maxwell's EM theory, in 1905 with Einstein's light quanta, in 1917 with Einstein's stimulated emission, and in 1925 when G N Lewis coined the word photon. What has changed recently is the way of looking at things, terminology, methodology, the rate of the discovery of new phenomena which not only fit into this foundation but also buttress it, the way technology has developed out of it as never before, and the way ageold concepts are understood in their content both irreducibly and far more widely. Therefore, notwithstanding the optics revolution, curricula have not gone far beyond biprism fringes, Newton's rings and lens formula. Quantum mechanics, nuclear physics, solid state physics and electronics were incorporated into curricula without any problems because the basic principles had changed completely. In this paper I give selected examples of how topics could be taught differently. Modifications may be made depending on local conditions - background of students, availability of resource material, of specialized teachers, teaching level, etc. "New optics" is essentially "physical" optics but "geometrical" optics is as "physical" so we need a new term. The term "Fourier optics" emphasizes the mathematical rather than physical character so I use the name "superposition optics" (- the title of my forthcoming book).

\section{WHAT IS LIGHT}

What is light? The teaching of optics even in school must begin with what exactly is known about the nature of light. Being told that light is electromagnetic waves does not demand deep knowledge of $E M$ theory; suffice it to have students know that electric and magnetic fields exist. I am appalled that even today in UG courses in many universities light is called a "disturbance", not an electromagnetic field! Anyway we refer only to the electric field $\mathbf{E}=\mathbf{E}(\mathbf{r}, \mathbf{t})$ because most detectors are sensitive to it. This must be a solution of the Maxwell wave equation

$$
\nabla^{2} E-\frac{1}{c^{2}} \frac{\partial^{2} E}{\partial t^{2}}=0 \quad \text { or } \quad \frac{d^{2} E}{d z^{2}}-\frac{1}{c^{2}} \frac{d^{2} E}{d t^{2}}=0
$$

If $\mathbf{E}_{\mathrm{i}}$ are many light fields at a point $\mathrm{P}(x, y, z)$ the resultant field there is

$$
E_{\text {resultant }}=\sum_{i} \boldsymbol{E}_{i} \quad \text { or } \quad E_{\text {resultant }}=E_{1}+E_{2}+E_{3}+\cdots
$$

This is the superposition principle. In Figure $1(p 3)$ the independent readings in detectors $D_{1}, D_{2}$ taken with only the respective sources $\mathrm{S}_{1}, \mathrm{~S}_{2}$ operating would not alter if the two sources were to operate concurrently. Partitions $\mathrm{P}$ prevent light reaching the wrong detector. If a third detector were to be placed in the region $\mathrm{R}$ of overlap the reading would correspond to an electric field that is the sum (eq 2) of the two beams. The photon picture arises out of light-matter interaction which should immediately be discussed - not waiting for a subsequent course in "Modern Physics" - introducing stimulated absorption, spontaneous emission and stimulated emission by means of the following equations:

$$
\begin{aligned}
\text { Stimulated absorption: } & E_{\ell}+\hbar \omega=E_{u} \\
\text { Spontaneous emission: } & E_{u}-\hbar \omega=E_{\ell} \\
\text { Stimulated emission: } & E_{u}+\hbar \omega=E_{\ell}+2 \hbar \omega
\end{aligned}
$$

They are mathematically the same, viz $\mathrm{E}_{\mathrm{u}}-\mathrm{E}_{\ell}=\hbar \omega$, but are written to convey the physical idea of what is happening. It is customary to leave the concept of stimulated emission to later years; it is a mistake in view of the need to introduce lasers early enough.

The only way to reconcile the two pictures is to draw correspondences between them (Table 1). The earlier the students are told that these are two independent descriptions which cannot be put under one single concept the better it is because they do not get conditioned by just one picture for a long time before coming to the second. Similarly the earlier they are familiarized with vectors (for EM fields are vectors) and complex representation (for it is convenient) the better it is. Whatever is said using the complex notation can equally well be said with the cosine one. Yet another convenience is using angular frequency $\omega$ rather than frequency $v$, and wavevector $\mathbf{k}$ 
rather than the wavelength $\lambda$, once the relationships are established. Plane monochromatic solutions of the wave equation are written as

$$
\boldsymbol{E}=\boldsymbol{E}_{0} e^{j(\boldsymbol{k} \cdot \boldsymbol{r}-\omega t+\alpha)} \quad \text { or } \quad \boldsymbol{E}=\boldsymbol{E}_{0} \cos (\boldsymbol{k} \cdot \boldsymbol{r}-\omega t+\alpha)
$$

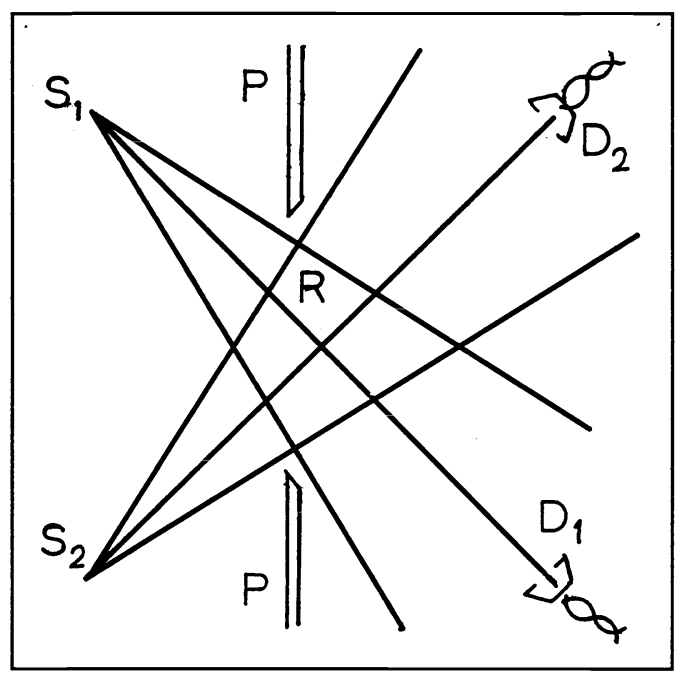

In the photon picture the observed quantity is energy difference which when divided by $\hbar$ yields one that is commensurate with frequency so we call it so; similarly momentum is the observed quantity which when divided by $\hbar$ yields what we may call "wavevector". It is good to introduce the correspondence of photon flux with irradiance right away. It is customary to define irradiance as being proportional to $\mathbf{E}^{2}$. It must be pointed out that no common detector of light including our eyes is sensitive to $\mathbf{E}$ directly since it cannot follow variations at $10^{14}-10^{15} \mathrm{~Hz}$. We deal with time-averages like $\left\langle\mathbf{E}^{2}\right\rangle . t$ drops out automatically by expressing irradiance as $\left\langle\mathbf{E}^{*} \cdot \mathbf{E}\right\rangle$. One must guard against taking squares or arbitrary products of complex quantities.

Figure 1 Principle of superposition

Table 1

Correspondence between the wave and photon descriptions

\begin{tabular}{|c|c|c|c|}
\hline wave picture & \multicolumn{2}{|c|}{ quantity } & photon picture \\
\hline$\omega$ & frequency & $\begin{array}{l}\text { energy } \\
\text { frequency }\end{array}$ & $\begin{array}{c}E_{u}-E_{l}=\Delta E \\
\omega=\Delta E / \hbar\end{array}$ \\
\hline$|\mathbf{k}|=\omega / \mathbf{c}$ & wavevector $\mathbf{k}$ & $\begin{array}{l}\text { momentum } \mathbf{p} \\
\text { wavevector } \mathbf{k}\end{array}$ & $\begin{array}{c}\mathbf{p}=\hbar \mathbf{k} \\
|\mathbf{k}|=\hbar \omega / \mathrm{c}\end{array}$ \\
\hline$I=\left\langle\mathbf{E}^{2}\right\rangle$ or $\mathbf{E}^{*} \cdot \mathbf{E}$ & irradiance & flux & N/At \\
\hline $\mathrm{R}=E_{x} / E_{y}$ & polstate & spin-p relation & $\begin{array}{c}\quad e g: \mathbf{S} \| \mathbf{p} \\
\text { is left circpol }\end{array}$ \\
\hline $\begin{array}{l}\gamma=\left(\mathrm{I}_{\mathrm{x}}-\mathrm{I}_{\mathrm{n}}\right) /\left(\mathrm{I}_{\mathrm{x}}+\mathrm{I}_{\mathrm{n}}\right) \\
\left(\mathrm{I}_{\mathrm{x}} \equiv \max , \mathrm{I}_{\mathrm{n}} \equiv \min \right)\end{array}$ & $\begin{array}{c}\text { (coherence) } \\
\text { contrast }\end{array}$ & coherence & $\begin{array}{l}\text { all } s_{i} \text { are } \| \\
\text { all } \mathbf{p}_{\mathrm{i}} \text { are } \|\end{array}$ \\
\hline
\end{tabular}

Much appears mysterious to students if not confusing, $e g$ the photon. In a diagram a wavy line with an arrowhead is marked by the magic term $\hbar \omega$. What does it stand for? Consider a $1 \mathrm{ps}$ pulse from an $\mathrm{Ar}^{+}$laser for which $\lambda=514.4 \mathrm{~nm}, v=5.83 \times 10^{14} \mathrm{~Hz}$. Its spectral width $^{3}$ is at least $10^{12} \mathrm{~Hz}$. Then $v$ loses its original "monochromatic" meaning! So we make the photon a wave packet, a coherent

\footnotetext{
${ }^{3}$ See $\S 4(5)$ below.
} 
superposition of monochromatic waves - but then it does not have a single frequency. For superposition we have to deal with different amplitudes and phases so the picture seems hopeless. For curricular purposes therefore the right way is to assert that the two pictures cannot be amalgamated. Readers who wish to have further erudition on this may refer to late G W Series' excellent article in Current science (1993 $65 \mathrm{Nr} 8$ 599-604) which also points out that it is spontaneous emission that is difficult to understand, not stimulated absorption and stimulated emission. My own experience is the same.

\section{COHERENCE}

The concept of coherence so central to all optics is the one most neglected. It comes directly from experiments, it does not fall out of an equation. There is no better way to introduce it than to perform actual and thought experiments with the Michelson interferometer, that most elegant and fascinating of instruments but which BSc students never come across and MSc students may barely do so. It deserves to be taught in its own right, not just because the Michelson-Morley experiment was done with it. Let an HeNe laser illuminate the instrument (Figure 2). The irradiance $I$ is measured at point

$$
\begin{aligned}
\delta=2 k d & =p 2 \pi \text { for } I_{x} \text { (maximum) } \\
& =\left(p+\frac{1}{2}\right) \pi \text { for } I_{n} \text { (minimum) }
\end{aligned}
$$

D. The incidence being only normal to the mirrors the geometry is easy. The conditions for irradiance maxima and minima are

I have set up a student experiment using a photodiode to measure $I$, a motorized drive to turn the screw and an $x y$-recorder to plot a graph of irradiance $v s$ the path difference $2 d, d$ being the mirror separation (Figure 3, $p 5$ ). From this the contrast, $\gamma$ of Table 1 , is calculated and plotted against $2 d$ (Figure $4, p 5$ ). In the case of the laser the curve marked $\mathrm{L}$ is obtained and the others for other sources. The experiment is not too easy with tha latter but the results of a thought experiment given in the form of Figure 4 are easily understood by students. Several conclusions are drawn from this experiment:

(1) The beams from the two mirrors combining at D give variable irradiance depending on $2 d$ so the result of their superposition is obviously "more than the arithmetic sum of its parts". This is the synergy of interference.

(2) With laser light the contrast, say with $2 d$ not too small, still has the highest value (unity, here) so there is something special about laser light. Its waves seem to be "smooth", not "gusty" like those of the other sources.

(3) Thus the experiment gives a clear concept of "smoothness" which we shall call coherence. With laser light the contrast remains very nearly unity for quite large values of $2 d$; let the largest value be called "coherence length" $d_{\text {coh }}=2 d_{\max }$ from which we get the coherence time $d_{\mathrm{coh}} / \mathrm{c}$ over which the waves are smooth. It is infinite for an ideal laser, quite long for actual lasers and quite small, $\approx 10^{-10} \mathrm{~s}$, for ordinary sources indicating that their waves are not smooth.

(4) Since there is a time-involvement we call this temporal coherence. The simple quantity "contrast" (Michelson's "visibility") is a measure of it. For coherent sources it has the value unity, for incoherent sources zero and for most sources something in between, indicating that the last may be called partially coherent.

(5) To view "spectra", $i$ e curves of the amplitude $\mathrm{A}(\omega) v s \omega$, what do we do? For sodium light of wavelength $589 \mathrm{~nm}$ we have $d_{\text {coh }} \approx 20$ $\mathrm{cm}$ or $t_{\mathrm{coh}} \approx 7 \times 10^{-10} \mathrm{~s}$. Noting that $\Delta v=1 / \Delta t$ and $\lambda v=\mathrm{c}$, we have $\Delta \lambda=\lambda^{2} / d_{\mathrm{coh}} \approx 0.002 \mathrm{~nm}$. Figure 5 shows the spectrum of laser as well as sodium light.

Large pseudo-Michelson fringes on a wall are easy to show. A convex lens of $f=4 \mathrm{~mm}$ placed in the laser beam path gives sufficient range of angles at which the light floods the interferometer to have many fringes according to the Michelson equation $2 d g(\theta) \equiv 2 d \cos \theta=p \lambda$. A concave lens of suitable focal length placed between the interferometer and the wall expands the fringes 
further. As Figure $6(p$ ) shows, this arrangement can be used to evolve the concept of spatial coherence as well. From Figure 6 the condition for maxima is deduced as ( $c f 2 d \cos \theta=p \lambda$ of Michelson)

$$
2 d\left\{G\left(\theta_{1}, \theta_{2}\right)\right\} \equiv 2 d\left\{\sin \theta_{1}-\sin \theta_{2}\right\} /\left\{\sin \left(\theta_{1}-\theta_{2}\right)\right\}=p \lambda
$$

Or since $\theta_{1}, \theta_{2}$ are given by

$$
\tan \theta_{1}=r_{p} /(L+D-2 d) \text { and } \tan \theta_{2}=r_{p} /(L+D)
$$

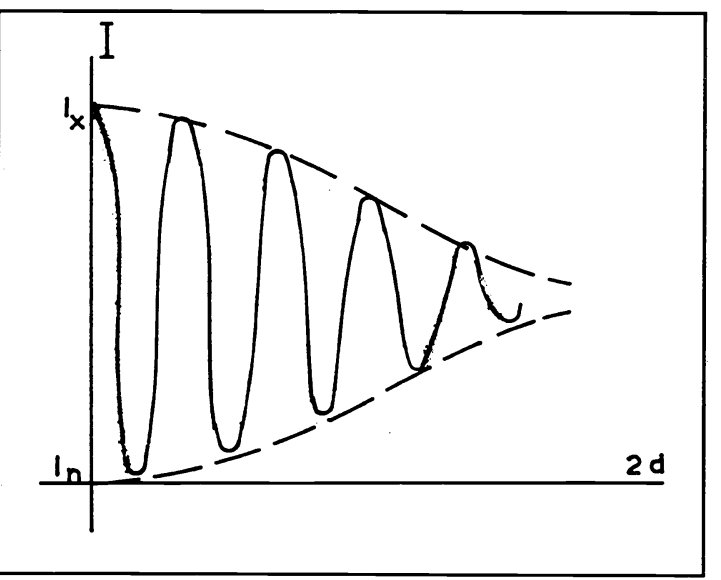

Figure 3 Irradiance variation

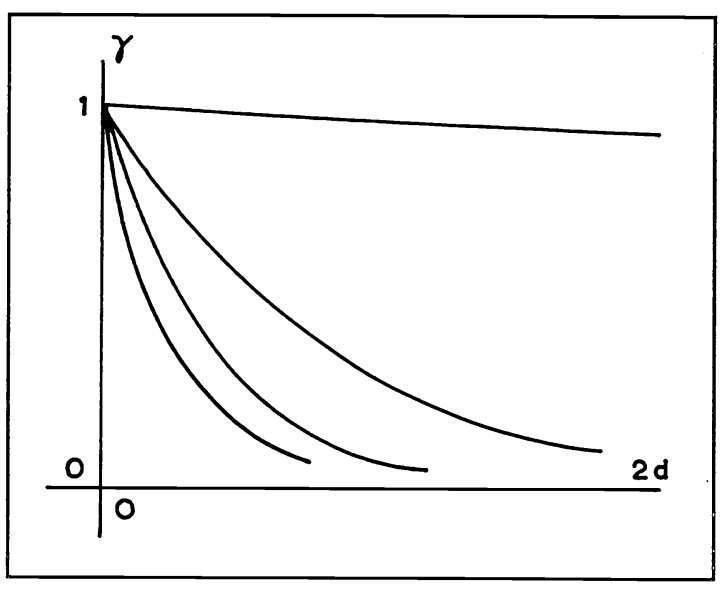

Figure 4 Contrast of central fringe

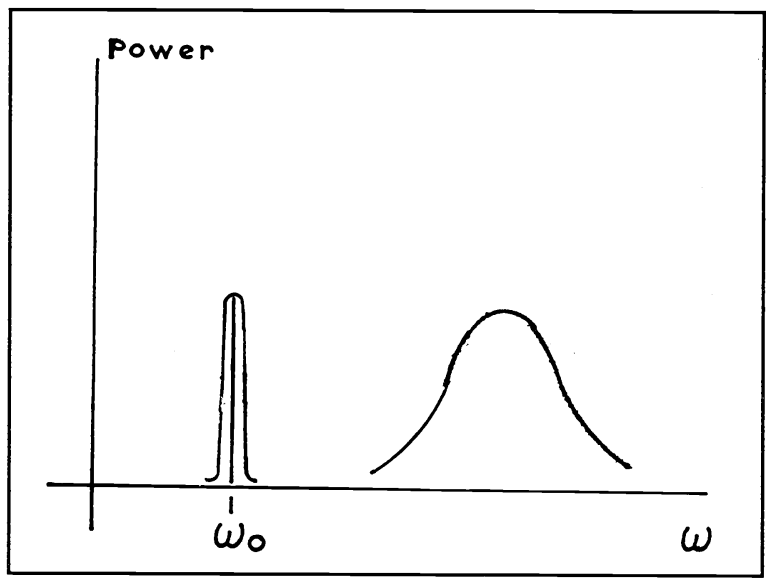

Figure 5 Frequency spectrum
$2 d \tilde{G}(L, p, d)=p \lambda$

we could write the condition for the maxima asThe radii of the rings, which are sections of real interference hyperboloids (unlike in the setup wherein a lens forms them), thus depend upon the positions of the beam expander and screen as well $f$. $\lambda$ is determined by making measurements which are more complicated than those of Michelson fringes at infinity. Another - usual - arrangement for studying spatial coherence is the Young experiment, or its modern Thompson-Wolf version. Temporal and spatial coherence represent the classical cases of "division of amplitude" and "division of wavefront" respectively. Thus I am saying that the concept of coherence comes from experimental observations and should be taught exactly for what it is, not out of the blue. The essence of teaching is to put ideas in the form of words and diagrams rather than

terrifying expressions of correlations. The teacher must innovate. 


\section{POLARIZATION I}

There is no simpler phenomenon than polarization to establish that $E M$ waves are vectorial so its teaching should precede rather than follow that of other topics. The most general $E M$ wave is most conveniently expressed as

$$
\boldsymbol{E}=\boldsymbol{E}(\boldsymbol{r}, t)=\boldsymbol{f}(\boldsymbol{r}) e^{j[g(\boldsymbol{r})-\omega t]} \text { or } \boldsymbol{f}(\boldsymbol{r}) \cos [g(\boldsymbol{r})-\omega t]
$$

This inhomogeneous wave - that is, in which the cophasal surfaces do not coincide with constant amplitude surfaces - is very complicated so we simplify it by demanding that $\mathbf{r}$ appear only in combination with a certain vector $\mathbf{k}$ as

$$
E(r, t)=E(k \cdot r, t)
$$

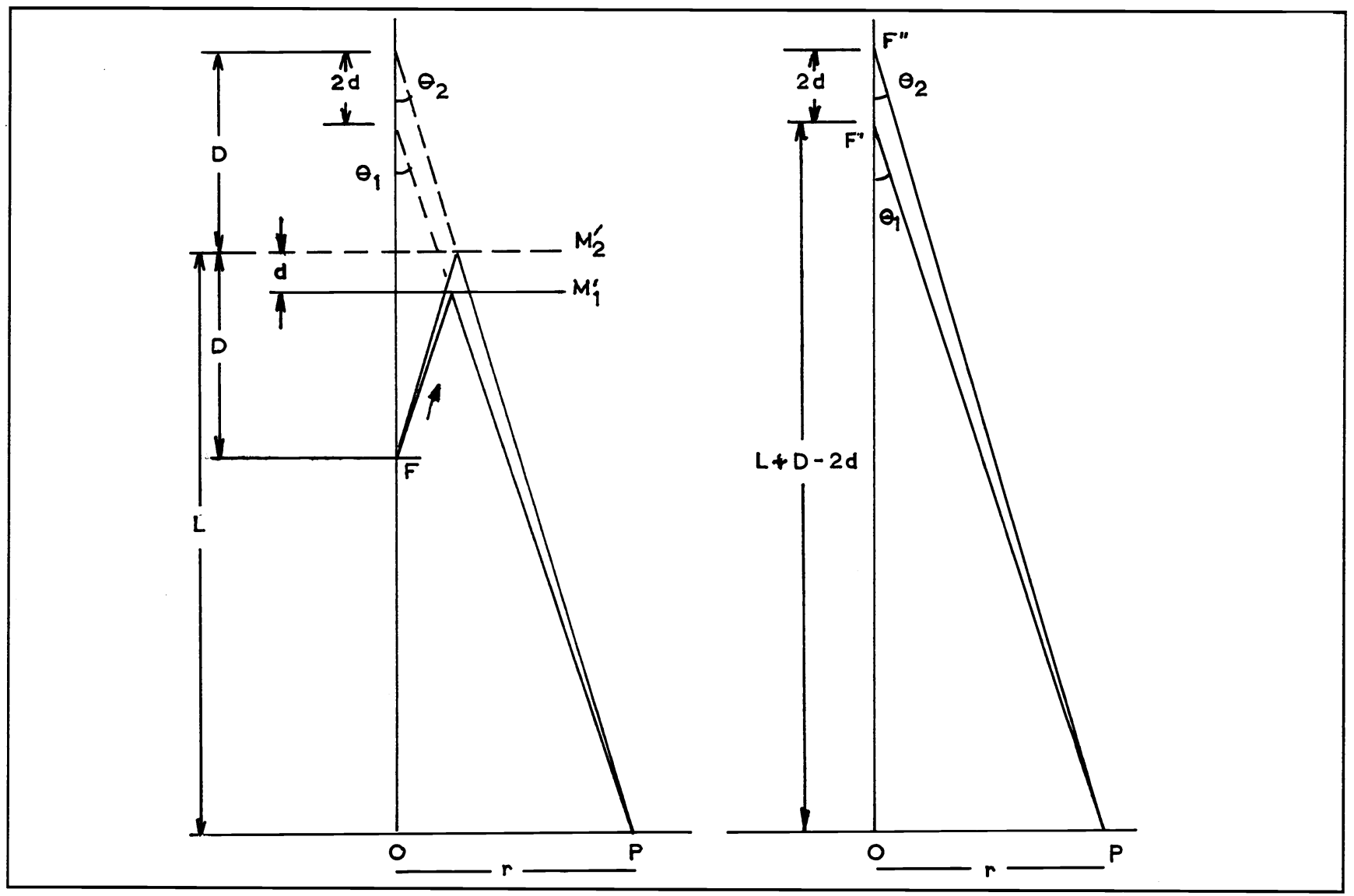

Figure 6 Developing the concept of spatial coherence, using the Michelson interferometer

This plane wave is simpler but the time-variation can still be complicated. So we think of "monochromatic" plane waves

$$
E(r, t)=E_{0} e^{j(k \cdot r-\omega t+\alpha)} \quad \text { or } \quad E_{0} \cos (k \cdot r-\omega t+\alpha)
$$


This is a pretty simple wave but even now $\mathbf{E}_{0}$ can vary. If we restrict the variation to simple rules the wave will be the simplest possible, viz polarized. If polarization is so simple a concept why is it a bugbear to students and teachers alike? The answer is that current teaching combines difficult crystal physics with the simple concept of polarization, making the latter difficult to understand. Crystal physics is interesting, beautiful and provides polarizing and retarding devices; but it is external to the concept of polarization, so the two topics be delinked. Crystal physics should follow polarization.

Let us restrict $\mathbf{E}_{0}$ to the plane perpendicular to $\mathbf{k}$ and choose the $z$-axis along $\mathbf{k}$. The $x$-and $y$-axes are chosen arbitrarily in the plane of $\mathbf{E}$ and $\mathbf{B}$. Denoting the phase difference between $E_{x}$ and $E_{y}$ by $\delta$ the electric field then is written as

$$
E(r, t)=i E_{x}+j E_{y}=\left[i A+j B e^{j k \delta}\right] e^{j(k z-\omega t)} \text { or } i A \cos (k z-\omega t)+j B \cos (k z-\omega t+\delta)
$$

Eliminating $t$ explicitly from these gives the polarization ellipse:

$$
E_{x}^{2} / A^{2}+E_{y}^{2} / B^{2}-2\left(E_{x} / A\right)\left(E_{y} / B\right) \cos \delta=\sin ^{2} \delta
$$

Deriving the special cases of linearly or circularly polarized light from this general ellipse is standard practice, the mathematics being easily understood. What seems not so easy is the physics of it. What does a polarized wave look like? Is the centre of the ellipse the source? What is left-handed and what right-handed? Are there other ways by which all this could be understood? What is the use of all this?... and such questions bother students and teachers alike, with the result that they get the feeling that the whole thing is best forgotten! Thus a beautiful part of optics is missed.

\section{POLARIZATION II}

Eq 13 needs to be interpreted properly. Time has really not been eliminated. What has been done is to sit at a point $z=z_{0}$ and look at the light wave as it comes along. We have, as it were, taken a section of space at that point so we term this diagram of an ellipse a "sectional pattern". It tells us how $\mathbf{E}\left[z=z_{0}\right](t)$ varies. The usual special cases are obtained by giving $\delta$ and the ratio B/A different values (Table 2). Some people write the phase as $\omega t$-kz in which case the sign of $\delta$ changes and "left" and "right" of this table get

Table 2

\begin{tabular}{|c|c|c|c|c|}
\hline$\delta$ & $\mathbf{B} / \mathbf{A}$ & polstate & description & handedness \\
\hline 0 & arbitrary & linpol & $\mathbf{E}$ at acute angle to $O x$ & - \\
\hline$\pi / 2$ & $\begin{array}{c}\text { arbitrary } \\
\mathrm{A}=\mathrm{B}\end{array}$ & $\begin{array}{l}\text { ellipol } \\
\text { circpol } \\
\end{array}$ & $\begin{array}{l}\text { ellipse: axes } \| O x, O y \\
\text { circle }\end{array}$ & $\begin{array}{l}\text { left } \\
\text { left }\end{array}$ \\
\hline $0<\delta<\pi$ & arbitrary & ellipol & ellipse at angle to $O x$ & left \\
\hline$\pi$ & arbitrary & linpol & $\mathbf{E}$ at obtuse angle to $O x$ & - \\
\hline $3 \pi / 2$ & $\begin{array}{c}\text { arbitrary } \\
\mathrm{A}=\mathrm{B}\end{array}$ & $\begin{array}{l}\text { ellipol } \\
\text { circpol } \\
\end{array}$ & $\begin{array}{c}\text { ellipse: axes } \| O x, O y \\
\text { circle }\end{array}$ & $\begin{array}{l}\text { right } \\
\text { right }\end{array}$ \\
\hline$\pi<\delta<2 \pi$ & arbitrary & ellipol & ellipse at angle to $O x$ & right \\
\hline
\end{tabular}

Different polarization forms

interchanged. This is minor compared to the confusion that arises if the convention of sitting at the light source is used; here we have used the one of looking at the source because that is the way experiments are done. The confusion is: Does "counterclockwise" 
correspond to "left" or "right"? The sense of rotation in a diagram, i e clockwise or counterclockwise, gets reversed but a left-handed wave remains left-handed whether it is looked at from the source or the detector.

The easiest way to see this is to plot the "snapshot pattern" - that is, with $t=$ fixed, say 0 - for a given wave ( $i e$ for given values of $\mathrm{A}, \mathrm{B}$ and $\delta$ ). Let $\delta$ be $\pi / 6$. Then at $t=0$ the component waves are

$$
E_{x}=A \cos k z, \quad E_{y}=B \cos (k z+\pi / 6)
$$

It is sufficient to plot $\mathbf{E}$ at $z=0$ and, say, $z=\pi / 6 \mathrm{k}$, as in Figure 7a. The helicity of the helix does not change by looking at it one way or the other. A similar exercise done for the sectional pattern will tell that, with the conventions used here, "counterclockwise" corresponds to left-handedness (Figure 7b). Plotting the polarization helix is completely independent of conventions, unlike the more usual sectional pattern, so it helps understand handedness.

The ratio $\mathrm{R}$ introduced in Table 1 is real for linpol, complex for ellipol and imaginary for circpol light ( $\pm i$ for left and right respectively). If it is required to refer the ellipse to its principal axes when they are not parallel to the coordinate axes standard transformations of coordinate geometry may be used. The uniqueness and simplicity of the two circpolforms also needs to be pointed out. Depending on the level, Jones and Mueller matrices may be introduced.

\section{Polarization III}

Polarized light exists in nature and can be synthesized too. The wire grid polarizer that when illuminated by completely unpolarized light has an output that is almost completely polarized normal to the wires. (The width and separation of wires are such (both $\wedge$ ) that we need not be concerned with blocking and diffraction problems.) This is easily understood on the basis of $i^{2} R$ Joule heating by induced currents. The functioning of polaroids is similar, although on a molecular level. During manufacturing polymer material is stretched, so that its long chain molecules are preferentially oriented in that direction, and exposed to iodine vapour. Such iodine atoms as are absorbed sit at the ends of side groups thus imparting to the material a small but finite $p$-type electrical conductivity. The transmission axis is normal to the molecules. My experience is that even school students understand all this. It is not necessary to mystify polarization.
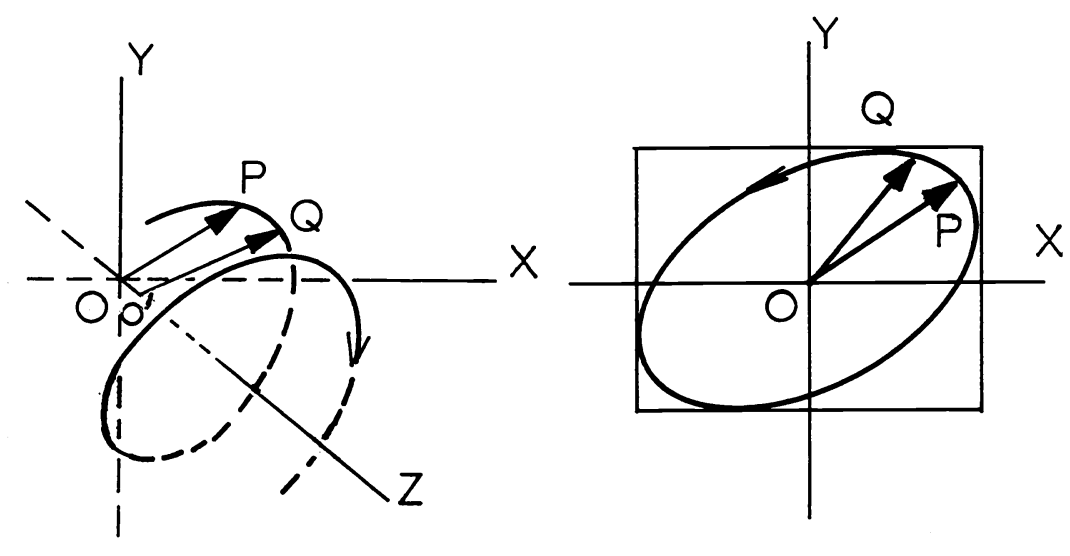

Figure 7 (a) Polarization helix, (b) Polarization ellipse
The Brewster phenomenon should be looked at from the viewpoint discussed in Sommerfeld's Optics, that it must take place if light is indeed $E M$ waves and this conclusion is reached without going into the details of the interaction of light with the atoms of the dielectric which in any case is difficult. Second, even opaque dielectrics show the phenomenon so a refractive index $n=\arctan \theta_{B}$ can be defined; then what is the meaning of refractive index? This indicates a possible generalization of the concept of refractive index. If light reflected from metal surfaces is studied for polarization states we have a further generalization that admits of a complex refractive index. It is not difficult to put these matters in simple terms. The point is that it should be done. 


\section{CRYSTAL OPTICS}

All of crystal optics can be derived from the Maxwell equations but it is best introduced by establishing certain observed properties of anisotropic materials. The best part of this methodology is that these phenomena can be demonstrated while talking about them. No more equipment than a large black sheet with a $3 \mathrm{~mm}$ round hole at the centre, a calcite crystal, two polaroids, a retarder made from layers of cellotape and an overhead projector is required to establish the following.

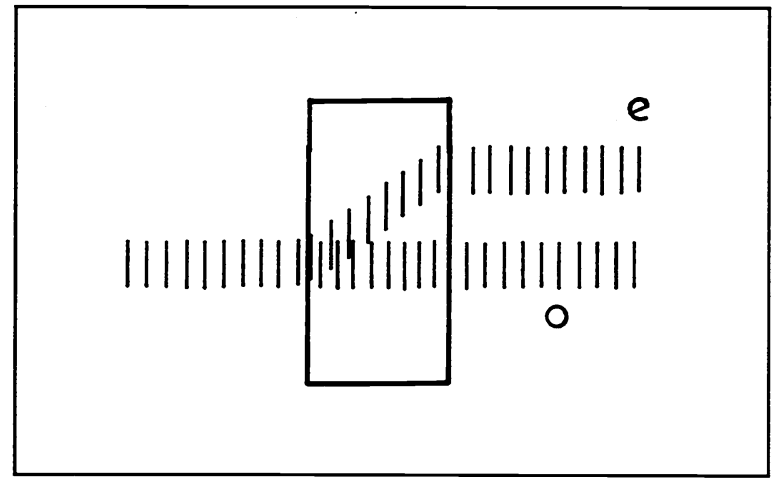

\section{Figure 8 Birefringence}

(a) Two invariant beams: A given slice of anisotropic material divides an incident wave into two waves which are distinguished by their independent speeds, polforms and directions of energy travel and are invariant with respect to the incident polform. In general it is not possible to have just one wave propagating. The two waves can actually be spatially separated by confining the incident wave to a narrow beam (Figure 8) rather than having it flood the entire face. An identically oriented slice of a different thickness will alter the phases of the two waves but not the respective speeds and polforms.

(b) Non-spherical wave surfaces and oblique energy flow: The wavefronts from an imaginary point source inside an isotropic medium will be spheres. A small portion of a wavefront travels along the radius vector joining the source to that portion along which also is the local

energy flow. The wave normal, $i e$ the direction of phase travel, coincides with the ray, $i e$ the direction of energy travel ${ }^{4}$. In anisotropic materials the situation is different (Figure 9, $p$ 10). The polar diagram of the ray (or phase) velocity vector is a two-sheeted surface, a sphere and an ellipsoid of revolution touching each other at two opposite points. We call them ordinary and extraordinary sheets respectively for obvious reasons. The oblique energy travel of the $e$-ray seems mysterious. The distinction between phase travel and energy travel is clarified by analogy with a row of persons who all walk with the same speed. If each walks normal to the row the situation corresponds to the ordinary wave. If each walks at the same speed and the same angle to the row the row advances parallel to itself but at a different speed and we have phase velocity and ray velocity.

(c) Different velocities of $o$ - and $e$-rays: The velocity $\mathbf{v}$ of $e$-ray travel depends on the direction of travel (Figure 9) ${ }^{5}$. Similar polar diagrams may be drawn for the velocity $\mathbf{u}$ of wavefront travel. $u_{o}$ is constant. $u_{e}{ }^{\prime}$ varies between $u_{o}$ and $u_{e}$. $(f)$ below gives the relation of $\mathbf{u}$ to $\mathbf{v}$. The respective refractive indices are given by $n_{o} \equiv c / u_{o}$, and $n_{e}{ }^{\prime} \equiv c / u_{e}{ }^{\prime}$ which is in the range of $n_{e} \equiv c / u_{e}$ and $n_{o}$.

(d) Double refraction: The property of the material leading to the decomposition of an incident wave - except along the line joining the points of tangency of the two sheets of the ray surface, called the optic axis ${ }^{6}$ - is called birefringence. Figures 10 a,b,c $(p 10)$ depict three differently cut sections of a crystal placed on a dot marked on paper which is looked at from above. Only one image is seen in the case $b$ because there is no birefringence along the line of sight. In the case $a$ the two images are spatially separated as well as in depth whereas in the case $c$ they are separated in depth only. Students should look at such three sections. No amount of description can ever replace the excitement of actually seeing two dots. For any given section the difference $\Delta n \equiv\left|n_{o}-n_{e}\right|$ is a measure of the birefringence for light travelling normally.

(e) Complete polarization: Using a linear polarizer it is easily found that each of the two waves is completely polarized linearly. Measurements show that the degree of polarization is $100 \%$.

\footnotetext{
${ }^{4}$ The practical visualization of a ray is the local direction of energy travel, not the wave normal.

$s$ A better way to say is that $\mathbf{v}$ depends on the direction of the electric field (that is, its polarization), which description gives the optical indicatrix. However, in the beginning it is not worth while going into it.

${ }^{6}$ Note that it is not necessary to refer to the external shape or angles of a calcite or quartz crystal in order to introduce the concept of the optic axis. The relation of the optic axis to such characteristics is not simple in most anisotropic materials.
} 


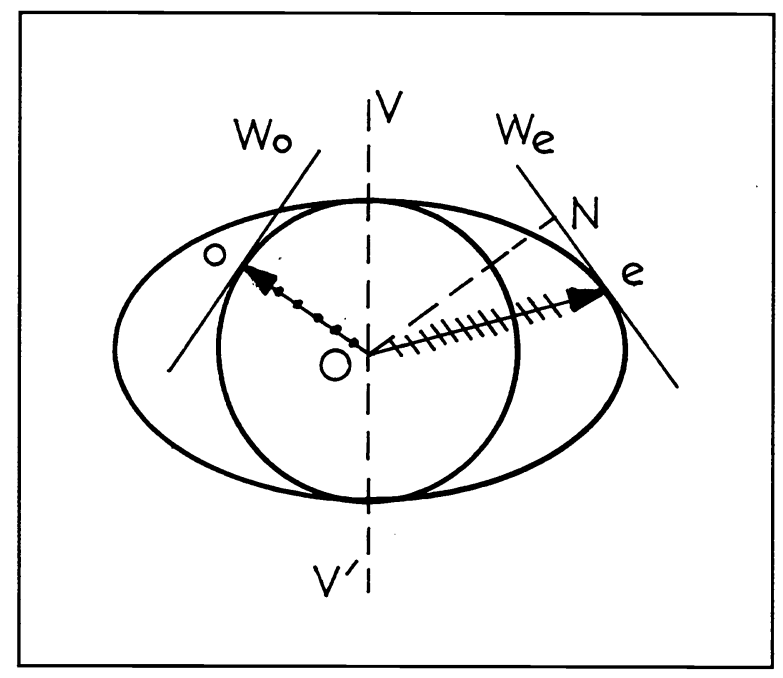

(f) Orthogonal polforms of o- and e-rays: The observation (e) above also establishes that the two polforms are orthogonal. How to find their vibration directions is also shown in Figure 9 in which $O N$ is proportional to $u_{e}$ and Oe to $v_{e}$. The light energy emitted by the fictitious source at $\mathrm{O}$ has, after a lapse of some time, arrived at $\mathrm{e}$ in the direction $\mathrm{Oe}$, not at $\mathrm{N}$ in the direction $\mathrm{N}$. The point $\mathrm{N}$ is obtainable from the ellipsoidal ray surface but the ray surface does not represent a wavefront emanating from $\mathrm{O}$.

These observations with due elaboration include all of uniaxial crystal optics needed at this stage. It is important to convey that birefringent materials provide polarizers and retarders, and how they work. The working of the Wollaston beam-splitter and Glan-Foucault polarizer is straightforward and easily understandable.

Figure 9 Two ray surfaces

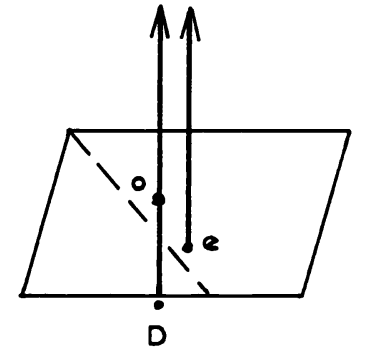

(a)

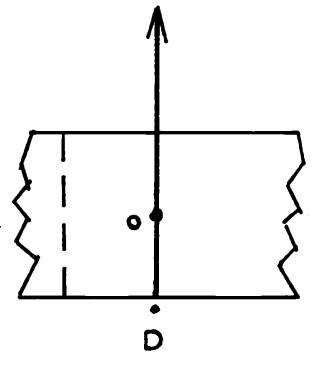

(b)

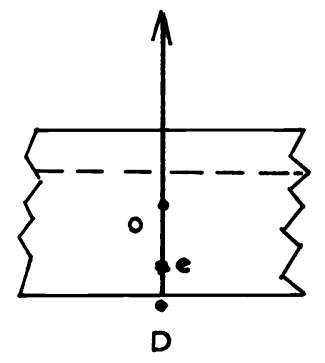

(c)

\section{Figure 10 Double refraction}

A retarder transforms an existing polform, it is a polform converter. If a crystal plate of birefringence $\Delta n$ and thickness $d$ transmitsan incident linearly polarized plane monochromatic wave of wavevector $\mathbf{k}$ the retardance is $\delta=k(\Delta n) d$. If the angle between the electric field and the fast axis is $\theta$ the amplitudes of the fast and slow components are $A \cos \theta$ and $A \sin \theta$ respectively. The transmitted wave is therefore elliptically polarized. A quarter wave retarder with $\delta=\pi / 2$ is a very convenient polform converter and should be demonstrated by taking suitable special values of $\theta$. Students should not be burdened with the relationship of the characteristic axes of a given crystal plate, viz "fast" and "slow", along the two linear vibrations $o$ and $e$, implied in $(f)$ above, with the optic axis and with "which is which". They are opposite, as it were, in calcite and quartz. The point is that any section of an anisotropic material except that normal to the optic axis has these orthogonal axes. Further, no matter how the subject is theorized, it is absolutely essential to have students do experiments on retarders. Only then will an understanding of retardation, polarization ellipse, handedness, etc develop. Practical uses of all this should be mentioned, otherwise students do not see the relevance. This remark is well taken for all teaching!

Many teachers, hence their students, associate polarization with calcite crystals and retarders and so on. This should be avoided. Many natural situations involve polarized or partially polarized light. Skylight is predominantly polarized linearly normal to the plane of the 
sun and the line of sight. Radiation from distant galaxies is polarized. A glowing hot sheet of, say, tungsten observed at grazing angles radiates light that is polarized normal to the plane of the sheet. Synchrotron radiation is predominantly polarized. In Zeeman effect all components are completely polarized in some way or another. Light reflected at Brewster angle from dielectrics - including household objects, wood, cement, paper, walls, foliage, tar road, pate of a bald person, etc - is pretty completely polarized. It is worth while to have students actually find this out by using polaroids. Crystals are not the only anisotropic materials. Some layers of toughened windscreens of cars act as polarizers, others as retarders, displaying beautiful bands of colours in daylight, the bands showing regions of equal stress - an excellent introduction to photoelasticity. More brilliant colours are seen by viewing through polaroids.

\section{PHASE AND GROUP VELOCITIES}

Waves are important because they carry and transport energy so let us examine this. If we consider the values of $z$ where $\phi \equiv k z-\omega t$ has a given value $\phi_{0}$ and wish to know at what points the phase has this same value we differentiate with respect to $t$ and get

$$
\frac{d \phi_{0}}{d t}=0=k \frac{d z}{d t}-\omega \text { from which } \frac{d z}{d t}=\frac{\omega}{k} \equiv v
$$

as the "velocity of propagation". In the case of plane monochromatic waves, whatever is propagated it is not energy. The excitation corresponding to the wave exists in all space for all time. It is a quasi-static situation, nothing being transported across any point except phase 7 . The velocity $v$ (or $c$ in vacuum) defined as the "velocity of propagation" has an unambiguous meaning only for the very simple waves like plane monochromatic waves, not otherwise. For example, the wave equation admits standing wave solutions but then $v$ does not have that meaning. Thus cosinusoidal waves do not transport energy. To see what the statement "waves transport energy" means we must look at something else. Let us add two waves of slightly different frequencies and get the usual wave groups of Lord Rayleigh:

$$
\begin{gathered}
\left.E_{1,2}=A \exp \{[k \pm(\Delta k) / 2] z-[\omega \pm(\Delta \omega) / 2) t]\right\} \text { or } A \cos \{[k \pm(\Delta k) / 2] z-[\omega \pm(\Delta \omega) / 2] t\} \\
E_{1}+E_{2}=2 A \cos \frac{1}{2}[(\Delta k) z-(\Delta \omega) t] \exp (k z-\omega t) \text { or } 2 A \cos \frac{1}{2}[(\Delta k) z-(\Delta \omega) t] \cos (k z-\omega t)
\end{gathered}
$$

Although the question of "transport" of energy remains vague as before, there are at least practical situations like beats in acoustics wherein we get a feeling that "something is travelling". So we say that, in addition to the phase velocity, in the limit of $\Delta \omega$ and $\Delta \mathrm{k}$ tending to 0 a "group velocity" could be defined:

$$
v_{\phi}=\frac{\omega}{k} \quad \text { and } \quad v_{g}=\lim _{k \rightarrow 0}\left[\frac{\Delta \omega}{\Delta k}\right]-\frac{d \omega}{d k}
$$

There is a better way of looking at group velocity. In Figure 11 there is a certain inter-group separation. If there had been three waves the diagram as well as calculation would be complicated but one could say that the separation would be greater. For four waves it would be still greater. For a very large number of waves it would be so great as to be practically infinite in which case we speak of a "wave packet" (Figure 12, $p$ 12) and can at least say that in time $t$ the wave packet has travelled the distance $z$ or that its velocity is $z / t$. But what exactly is the wave packet? It is where all - or at least a very large number - of waves of differing frequencies have the same phase, say $\phi_{0}$, irrespective of frequency so that

\footnotetext{
${ }^{7}$ Among textbooks Sommerfeld's Optics is the only one that makes this point.
} 


$$
\frac{\partial \phi_{0}}{\partial \omega}=\frac{\partial(k z-\omega t)}{\partial \omega}=0 \quad \text { from which } \quad \frac{z}{t}=\frac{\partial \omega}{\partial k} \equiv v_{g}
$$

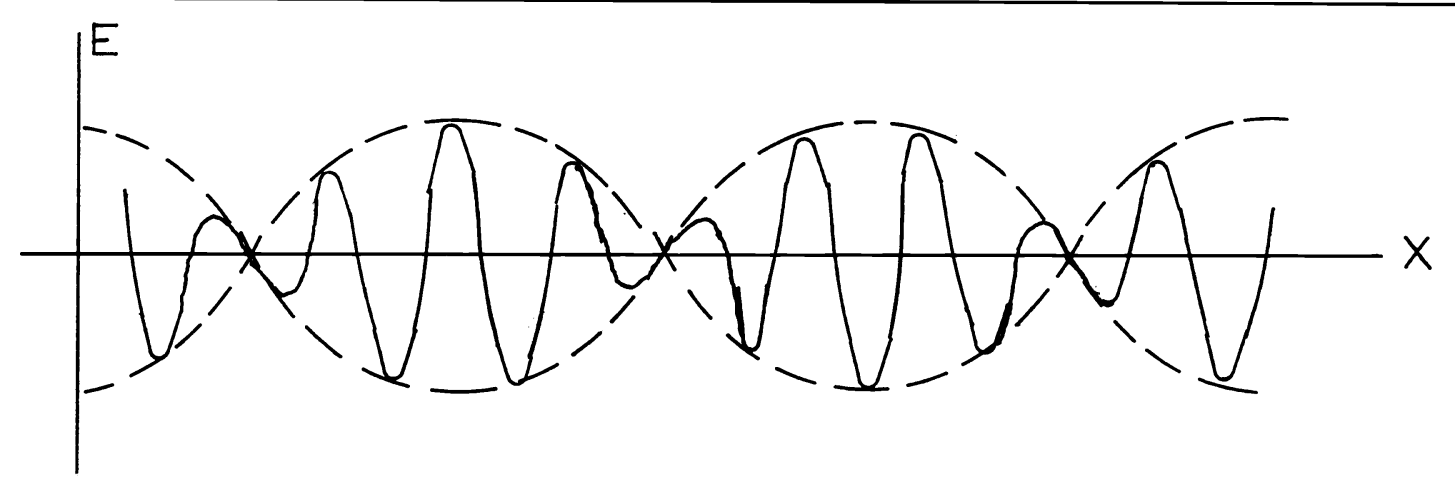

Figure 11 Rayleigh wave groups; beats

If the frequency is a function of $k$ only we can write

$$
v_{g}=\frac{d \omega}{d k}
$$

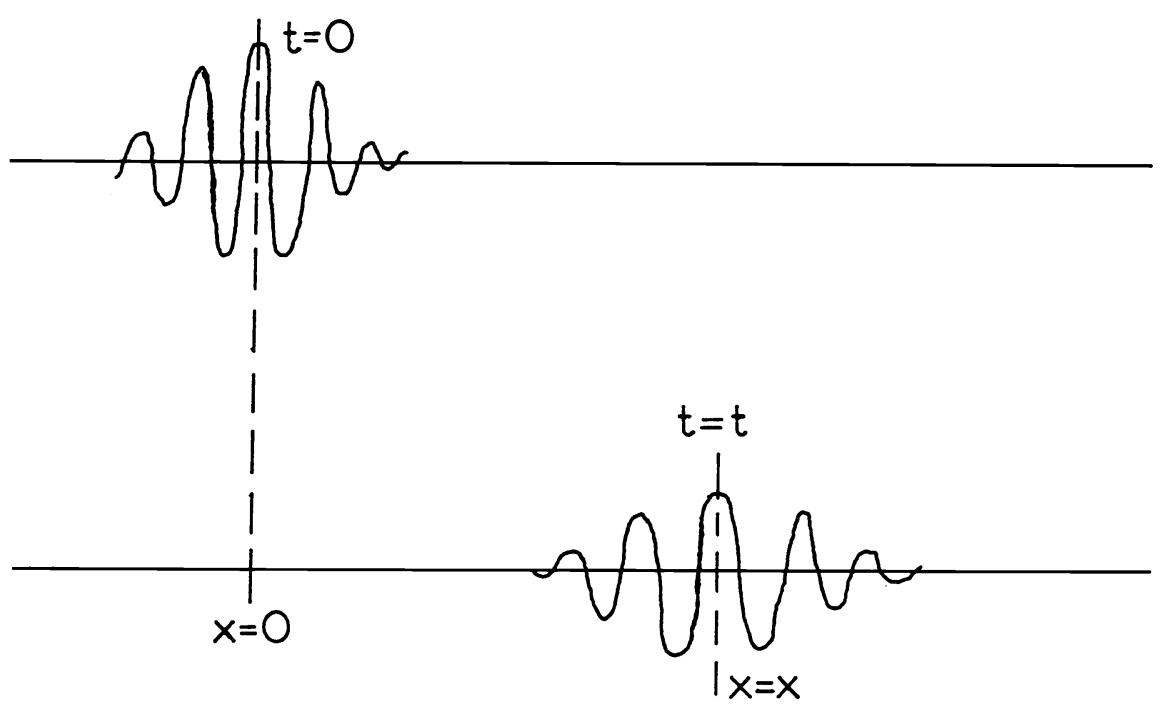

Figure 12 Calculation of group velocity

This is the meaning of "group velocity". This is the so-called "stationary phase approximation". Whereas students may not be frightened with this jargon the method is straightforward and easily understandable. R W Wood in his classic Physical Optics (3rdedreprinted by the Optical Society of America 1988, pp 21-23) has used a similar method. 


\section{DIFFRACTION}

Diffraction is natural whereas interference experiments are contrived. The basis of teaching is the Huygens principle, a term that be abandoned for it is not a separate principle of physics. It is merely a construction based on the principle of superposition. In its original form it is not even correct. The correct, $i e$ Fresnel-Kirchhoff, form differs in three respects: (1) obliquity factor, (2) dependence of the diffraction field on wavelength, (3) the phase of the superposed field being exactly $\pi / 2$ off that of the incident field. Finally, it is only an approximation. The teaching, therefore, needs to be drastically modified. The definition of diffraction is 'Diffraction is that non-rectilinear propagation of light that cannot be attributed to reflexion and refraction'. When light illuminates an aperture in an otherwise opaque screen what really happens is that its electromagnetic field sets into vibration the electrons of the material, say, of the jaws of a slit. They re-radiate into the space behind the slit. The coherent superposition of all such fields is the diffraction field. There is nothing at all in the space between the jaws to interact with the incident light field. True, Sommerfeld's exact solution of ninety years ago is too complicated to be given to any but the soulfully devoted students of optics. The point I am making is that these considerations need to be incorporated into the teaching of diffraction.

The single slit is followed with the diffraction grating that has a stepwise transmission: through a given strip of the grating the incident light is transmitted either fully or not at all. This corresponds to (1) a real absorption and (2) a transmission coefficient that can only take the values 0 and 1. In modern optics something more is required, viz, in general, complex transmittance function: not only is a part of the incident wave absorbed but also is the transmitted part retarded. The transmittance function for a two-dimensional aperture/obstacle may be written as

$$
\Theta(x, y)=T(x, y) \cdot e^{j \delta(x, y)}
$$

or in its cosine form, where $\delta$ is the retardance of the region around the point $(x, y)$. Indeed the idea is not new for the Michelson echelon gratings, unlike ordinary gratings, had imaginary transmittance function. These ideas are directly useful in holography which should definitely be taught at all levels including school. Teaching of diffraction is these days limited to Fraunhofer diffraction and that is a great pity because equally interesting and useful application, viz holography, is based on Fresnel diffraction. Constraints of time do restrict what is taught but as far as holography is concerned the interference pattern of concentric circles formed between a plane wave and a spherical wave, and subsequent Fresnel diffraction by it is all that is required to be taught. (For the methodology of teaching holography to undergraduate or school students reference may be made to A S Pārasnis, 1990, Basic principles of holography, Physics Education 7(3) 181-193.)

\section{EXPERIMENTS}

Students $d o$ find it boring to struggle with complicated apparatus in a dingy, smelly darkrooms, peering through eyepieces for hours and still not finding the fringes which the teacher said should be there. All that is left to them at the end of the day is to copy someone else's observations. This is not very creditable to the community of Physics teachers but a hard fact. The simple solution is to acquire a few $\mathrm{HeNe}$ lasers and build experiments around them. The topics of image formation, filtering and processing can very easily be built around the classical Abbé-Porter experiments.

\section{EPILOGUE}

My purpose here is to make a case for overhauling physics and optics curricula so as to prepare students for entering the fascinating field of modern optics and a case for demonstrating optical phenomena while teaching optics at any level (including $\mathrm{PhD!}$ ). I have given examples of how only small changes of emphasis enable better teaching to be effected, better background to be prepared and students to be excited about the subject. Most topics already exist in all syllabi. What I am urging is a change in the methodology of teaching. In particular, the traditional sequence of topics taught - interference, diffraction, polarization - needs to be exactly reversed! The strategy of making small changes obviates the need of upsetting the applecarts of any academic system. In a sense I have tried to market optics. Optics teaching can't wait much longer! 